\title{
DIGITALISATION VS. INFORMATIZATION: DIFFERENT APPROACHES TO GOVERNANCE TRANSFORMATION
}

\author{
Alois Paulin ${ }^{1}$
}

DOI: 10.24989/ocg.v331.21

\begin{abstract}
The twentieth century brought humanity radically new knowledge in form of electronics, informatics, and telecommunication technologies, which gave rise to cyberspace as a channel for data exchange, data storage, and as an enabler of new approaches to steering and controlling realworld systems. Thus-created new opportunities have been successfully deployed for the automation of processes in areas such as production, service provision, data exchange, navigation, logistics, etc., or for creating new possibilities through concepts of virtualisation, respectively. While this has led to radical transformations of paradigms in industry and free market service provision, the systems that make up modern states have been broadly spared of disruption by these technologies. Behind this backdrop, this contribution aims to discuss the differences between digitalization and informatization as two differing approaches to system transformation. The discussion is set in the context of societal governance, where digitalization is the main approach to modernisation. The focus on digitalisation and the lack of progress towards informatization in this field of interest is criticized, and the advantages of informatization are brought to attention.
\end{abstract}

\section{Introduction}

Contemporary buzzwords containing attributes such as "Smart-*”, “*_4.0”, "Cyber-Physical”, "intelligent", etc., which dominate discussions in areas of sales, marketing, and consultancy (and as such slop over to scholarly deliberations as well) have superseded a previous generation of buzzwords characterized by attributes such as "e-*" / "electronic", or "digital". Although none of these terms are clear technical terms, the swap of terminology nevertheless reflects a change of the underlying matters at stake and is justified by existing advances in technology.

Amongst these buzzwords, the "4.0" suffix stands out in a special way. The suffix emerged as part of "Industrie 4.0", a high-tech strategy document of the German federal government from 2013 [6]. The objective of that document was to provide a future vision of industry by the year 2025, taking emerging trends and advances in Internet technology as a point of departure. In that document, "Industry 4.0" stands for the use of Cyber-Physical Systems (CPS) for industrial manufacturing, whereby a CPS according that document is a network of embedded computers, which can be used to individually steer manufacturing processes. CPS there is explicitly used as a synonym for the Internet of Things (IoT) [6]. In a nutshell: the German Federal Government through this document conveyed a vision that the Internet of Things will lead to a fourth industrial revolution (hence, the "4.0"), which will catapult German and the Western industry again miles ahead of the emancipated Asian competition.

\footnotetext{
${ }^{1}$ Facult of Organisation Studies in Novo mesto, Novo mesto, Slovenia
} 
The "4.0" buzz-suffix was soon picked up by other domains: Health 4.0 [31], Public Administration 4.0 [27], are two examples that have been taken serious enough to enter the scholarly discourse. This led scholars to re-adjust the "4.0" suffix in the context of the respective domains. While originally Industry 4.0 refers to an emerging 4th industrial revolution (the first one triggered by steam power, 2nd: introduction of the conveyor belt, $3 \mathrm{rd}$ : electronics, 4 th: digitalization), the " 4.0 " in other domains refers to a different kind of change. In Public Administration the " 4.0 " is interpreted as a futuristic administration that is fully automated, transparent, and non-political [27], while the "4.0" in Health refers to a future evolution of public healthcare systems where harvesting and governance of personal data plays a decisive role [18]. What is common to the use of "4.0" throughout these disciplines is that it refers to a novel generation in the respective domain.

This article aims to unravel the buzzwords and describe the underlying novelty of the switch to a new set of terminology. Section 2 shall describe the four generations of controlling structure, to interpret how the new terms came to be. There, the difference between digitalization and informatization shall be explained. Section 3 will discuss how digitalization and informatization translate to technology applied to control and deliver public governance. Section 4 will conclude with a discussion on the implications of informatization on public governance and outline the challenges for the scholarly debate.

\section{Understanding the four generations of controlling structure}

In order to understand the potential effects technology can have on industry, commerce, society, and societal governance, one must develop an understanding for the respective characteristics of changed approaches to technology. For this purpose, the "four-revolutions" model as popularly used in history and economics however is insufficient: While the four industrial revolutions (the last one yet waiting to happen!) may serve as milestones to segment evolution of society into characteristic areas of time in which a number of factors triggered a chain reaction of memorable societal changes (de-feudalisation, urbanisation, shuffles in economic and political power, changes in moral and societal values, ...), they focus on fashions of production (e.g. conveyor belt and automation as facilitators of mass production), availability of new technical systems (e.g. cars, railroads, telecommunications, bicycles), or materials (iron, petroleum, paper) [29], which are categories too broad to identify technologies that can trigger further transformations.

An approach to identify technology triggers is the generations model $[18,19]$. In that model, the focus of observation is the approach to controlling structured processes of work / production, where four distinct generations are identified: mechanization, automation, computerization / digitalization, and informatization. Unlike the revolutions model, which deals with historic time spans, the generations model focuses on the ripeness of a specific domain, and its influence on fostering progress in others.

The 1st generation (mechanization) refers to the introduction of machines in work processes. An example for this step is the 18th-century invention of the Jacquard loom as a game-changer in the production of woven fabric [12]. The Jacquard loom was a mechanical, man-powered device, which structured the process of weaving, and used a system of punch-cards to describe the weaving pattern. Other examples of structured, mechanized systems, are the late 18th-century threshing machine, or the 19th-century typewriter. Characteristic for the first generation is thus the transformation of previously unstructured work (like weaving, threshing, or writing) into a wellstructured, mechanized (i.e., conducted by machines) process, with manual labour used to progress through the individual stages of the process. 
The second generation (automation) is then characterized by the introduction of power to automate previously structured and mechanized processes. Instead of the individual stages of the process being moved manually, the process is now progressed automatically stage-by-stage without manual intervention. The 18th-century steam engine, the 19th-century electric Jacquard loom, or the late 19th-century Benz motor car, are all examples of automated machines belonging to the second generation of structure control, and so are 16th-century mechanical watches. The given examples, most of which coincide with the society-transforming industrial revolutions, must however not be misunderstood: automation of machines and devices has been known long before the renaissance of Western high civilisation [2]. Furthermore, automation is not limited to a specific type of power provided to the system - the spring, which powers a watch, the water stream that powered mechanical theatres (cf. [26]), the steam engine and the combustion engine as generators of power, and electricity, are all equally valid sources of power that enable automation.

In the 3 rd (computerization \& digitalization) and 4th (informatization) generation, control of structure and processes relies on electronics and the possibilities derived from that. Following two sections shall deal with their characteristics and implications in more detail.

\subsection{The third generation: computerization \& digitalization}

The $20^{\text {th }}$-century brought the digital computer (cf. the 1920 ies Lehmer sieve as an early nonelectronic digital computer), and electronics as a radical novelty to human knowledge. From these soon first computerized systems for industrial production emerged, which used digital computers and electronics for controlling process flows. Electronics as a way to control systems can be steered by software, which in turn enables a never before possible amount of precision and new possibilities to control a technical system.

Software enables a type of control that goes beyond mere automation: Zuboff's 1980ies book In the Age of the Smart Machine describes with fascination how software, which is used to steer processes of computerized machines, keeps in memory the state of the thus steered process, which in turn enables this very same software to act based on knowledge of the state - Zuboff called this ability informating [32]. Her use of informating thus refers to the inherent context-awareness of systems which have been designed in such way that software not only steers their performance, but also generates, stores, and uses information about the context: "The programmable controller not only tells the machine what to do - imposing information that guides operation equipment - but also tells what the machine has done - translating the production process and making it visible." (ibid., p.10)

Zuboff's fascination for the duality of information technology is comprehensible if one takes into account her time, in which a radically novel generation for controlling structured processes emerged. However, from a 21th-century perspective, the fascination has faded away, as software controllers (and their inherent state-awareness) became a normality in engineering and management in the digital age. Zuboff's informated smart machine, which not only automates processes of production, as 2nd-generation machines were capable of, but is also aware of its own current state within the context, is thus a machine managed by 3rd-generation controllers, whose primary objective is to automate specific processes (such as soldering car parts, harvesting crops, calculating salaries, or counting votes), while the machine's state-awareness enables an unprecedented level of precision and complexity. 
The defining characteristic of the 3 rd generation of control is thus the use of the computer as a device, which processes data, acts upon information, and composes instructions to contextconsciously govern the process(es) of the system it controls. This level of control is best called computerization - such system is then computerized. Typical examples of computerized systems are industrial robots, automotive electronics, computer-steered domestic appliances (fridge, dishwasher, electric stove, air conditioning, ...), etc.

Many scholars instead of computerization use the semantically largely overlapping, if not fully synonymous term digitalization. The crux with these terms is that they lack precision: digitalization is frequently used to refer to the use of information and communication technologies for business / administration [11], and also the Oxford English Dictionary (OED) defines it in such way ("The adoption or increase in use of digital or computer technology by an organization, industry, country, etc." [17]); other sources however use it as a synonym for digitization (also Oxford Dictionary of English (ODE) defines it as such), an established technical term referring to the transformation of analogue signals (or real-world items) into a digital representation. On the other hand, computerization $^{2}$ implies a closer proximity to hardware-controlling software. Accordingly, I suggest following use: electronics in the car and dishwasher make them computerized systems, while the use of accounting software makes a business digitalized.

\subsection{Fourth generation: informatization}

Up till the 3rd generation, technical artefacts were designed to control processes for production and processing of goods (e.g. loom, combine harvester), to control administrative processes (e.g. census, accounting), or to steer the functioning of devices (e.g. clock, dishwasher). Up till then, the controller as a steering mechanism was an integral part of the system - i.e., the way the system behaved was determined by the mechanism which was part of the system itself. Thus, in first and second generations of control, the control mechanism and logic was defined by the physical architecture of the system's hardware; the third generation introduced the switch to software, but left the architecture (i.e. its functionality and program flow) of the pre-compiled software determine the functionality of the machine.

The defining novelty of the 4th generation lies in the reliance on the digital (computer) file as the descriptor of a system's (or digital object's, respectively) characteristics and state. More specifically, the type of file at stake is the type which can be exchanged, shared, edited. While the computer file as a concept to store a system's state is known since the 1950ies, it is only the later evolution of file systems as part of wide-spread computer operating systems, and files in form of standardized, open file types, which made the new generation happen.

The modern computer file constitutes digital objects in their serialized - that is, written down in digitally readable structure, form. Computer programmes, which make use of the file, deserialize the information and make use of data / information / instructions contained in the file - the way a given file is used, depends finally on the system it is used by. A file can then be a composition of graphic elements, a plug-in for a computer program, or a software library that extends functionality of a computer program. A PDF file for example can be composed by a digital artist (and edited by its peers) using desktop publishing software such as Adobe Illustrator - such file will then contain a

\footnotetext{
${ }^{2}$ OED: "The action or process of computerizing an organization, activity, etc.; the conversion of information, text, etc., into a form which can be stored or processed by computer." [16] - note the proximity to digitization!; ODE: "convert (a system, device, etc.) to be operated by computer: the advantages of computerized accounting."
} 
logical composition of graphics and text, which can be interpreted by other software to instruct display hardware to render the graphical composition on the computer screen. The very same file can then be transferred to a printing software, which will instruct printing hardware to create a tangible instance of the digital graphic. During all stages, the file from this example remains the original digital object, which can be created, edited, deserialized, shared, copied, transformed, rendered to a human-perceptible representation, etc. in a potentially indefinite number of ways.

In order to be able to instantly co-work on a file, networked work spaces (computers with the required software) are of advantage, amongst which the file can be shared. The sharing of files that contain virtual compositions (graphics, multimedia, ...), software systems / components (executable code), or other types of digital objects, enables the emergence of virtual co-productive communities, which rely on cyberspace as a gathering environment (cf. [24]). This very emergence of cyberspace in terms of a dimension for interaction, production, and creation of value (online services, etc.), is another enabler of the 4th generation for controlling structure. Although at the end of the day all interaction in cyberspace is nothing but the exchange of data between terminal equipment, interaction in cyberspace is so different in quality and complexity, that it must be distinguished from plain exchange of signals / data as it occurs in telephone calls or telemetric readings. Foreseeing the upcoming change of quality of information and communication technologies, Nora $\&$ Minc in the years of the emerging Internet coined the word telematics in their The Computerization of Society [28]. Although the word telematics (as well as the prefix tele-, as in television, or tele-voting) meanwhile went out of fashion, the justification for the then-new word telematics is a relevant indicator of substantial change in generation of technology.

The modern file is thus more than a mere representation of a system's state (as the early computer file was), and more than a set of processing instructions (as would be sufficient for purposes of automating and informating a system) - it is a genuine object, which exists natively in its digital form and only when interpreted by software descends from cyberspace to the physical world (in perceptible form as print-outs / products / visualisations / music / movies, ..., or actions such as e.g. granted access to resources, movements of robot arms, etc.).

To refer to such 4th-generation system, following vocabulary should be used:

- Informatizing (verb) stands for the creation (or conversion) of a system into a form, which crucially relies on digital objects (described through files).

- A system, which crucially relies on digital objects, is informatized (attribute).

- Informatization refers to the culture of engineering systems, which are informatized by design, and thus to the 4 th generation as such.

All words, except for informatization, I'm hereby coining specifically for the purpose to satisfy the need for demarcating the 4th generation from the 3rd. The word informatization is being in popular use already, whereby the OED defines it as "the adoption of information technology; computerization" [17]. The crux with this existing semantics however is, that thus a total of three different words (informatization, digitalization, computerization) all would stand for vaguely the same - namely the use of an ambiguous mixture of information technology (software), digital computers (software + hardware), and electronics (hardware) in a given context (or their introduction therein). For sake of professional clarity in the use of words, I hereby accordingly 
propose to use the terms informatizing / informatization / informatized solely in the context of 4thgeneration systems as described herein.

\subsection{Unravelling the buzzwords}

Researchers interested in understanding systems of societal governance, are used to deal with ambiguous semantics: bureaucracy, for example, can mean a social class (like aristocracy or clergy), a type of organisation, the system of public administration, or the administrative procedure (cf. [1] for a rigorous treatise on that). Likewise, governance has a myriad of meanings [3], so does democracy, and so on. Given this culture of unclearness, it does not surprise if computerization, digitalization, and informatization, all mean kind of the same in popular (and sadly, also in scholarly) discourse - that is: introducing technology to modernise the way business is conducted. In above sections, I undertook the humble attempt to identify semantic differences of these terms and assign them to different situations of use. I consider such differentiation crucial for the advancement of our scholarly field, as only precise terminology can help us properly identify or define challenges and potentials that involve or affect multiple disciplines.

Having clarified the terminology, we can then move to align the buzzwords according to these categories. In the introduction to this article, two groups of terms were identified: "smart", "4.0", "cyber-physical", and "intelligent" belong to one group, which refers to the systems that rely on the 4th-generation of control; the other group are "e", "electronic" and "digital". The line of reasoning is as follows: "4.0" as per definition of the ur-document [6] refers to the use of cyber-physical systems; the attributes "smart" and "intelligent" as used in the context of man-made systems (i.e., either technical systems such as smart phones, or systems of organisation such as smart cities) refer to the systems' reliance on information systems which crucially depend on shared digital objects, have optional network connectivity (to instantly exchange digital objects) and make use of platform systems (software stacks, such as e.g. Windows as operating system, which hosts the graphics software Adobe Illustrator, which can be used as a tool to create and edit graphic compositions stored as PDF files) that provide functionality to create / render / edit / reuse, etc. the digital objects (cf. $[8,30]$ for a better explanation of "smartness"). Such characteristics of "smart" / "intelligent" / "cyber-physical" systems allow them to be independently extended during run-time beyond the limitations as imposed by the original makers of the system. This very ability to be extended makes them go beyond the limited abilities of systems that would otherwise fall under the 3rd-generation of control.

The second group ("e" / "electronic" / "digital") denotes systems and software without the ability for co-creation. An electronic / digital system's functionality is thus limited to providing a specific pre-determined routine. An e-voting system for example is built to register voters, assure integrity and anonymity of the votes, and provide results in the end. Although a modern-day e-voting system will be built using systems and tools that belong to the range of 4th-generation systems, the e-voting system itself remains a 3rd-generation system, since it isn't enabled for co-creation / extension by its users. The "e" / "electronic" / "digital" thus stands for systems that are "electronic" / "digital" to the extent that they are controlled by software, without being designed to be extended / amended by its tech-savvy users. 


\section{Transforming governance - digitalization vs. informatization}

Radically new possibilities of technology inspired scholars of the 1970-90ies to think about new possibilities enabled by digitalization and beyond: Nora \& Minc's telematics (end-1970ies) describes a transition to informatization, enabled by networked computers; Ascott's 1980ies telematic art [cf. 28] is a future vision of telematics-enabled co-creation (distributed authorship), where authors would co-create works of art by means of networked robots, even if working thousands of miles away from each other; Zuboff's end-1980ies informating [32] describes information systems, which gain smartness through their ability to be state-conscious. While these early predecessors of informatization already rely on computer networks, co-creation, or computer memory for situation-aware action, they do not take into account yet the computer file / digital objects as the crucial enablers of informatized systems.

New possibilities brought by the 20th century, were soon followed by radical transformation of domains such as communication, logistics, publishing, entertainment, retail and advertising, public discourse, and other domains of economy and civilisation. The manifold novelties brought by information and communication technologies over the last decades (Internet, the Web, cellular networks, satellite navigation), enabled by electronics and digital computers, do not need to be explicitly listed for one to understand how significantly the mentioned domains differ in 2017 if compared to 1967 , or 1917 ! What is crucial to understand, is the leap of these domains from digitalization and informatization: Was the telefax yet a typical 3rd-generation terminal system for transmitting digitized letters, the email client is already an informatized system relying on the email as a container of digital objects. Was the GPS system yet a 3rd-generation constellation of manmade celestial objects for calculating one's position on the globe, Google Maps \&co. use the Internet and a system of accessible standards for co-creating an informatized ecosystem.

\subsection{Governance digitalization - a history drag?}

While the leap from digitalization to informatization radically transformed the world we live in, making old cultures and business models die out to leave room for new (think of paper maps vs. modern automotive navigation, phone boots vs. smart phones, physical journals vs. online papers, etc.), it failed to transform the systems of governance, which stuck in the 3rd generation of structure control. Even though politics, public administration, and the judiciary, as the key branches of public governance have undergone a substantial evolution by taking-up use of software to modernize their front- and back-office activities, this evolution has come to a stop at digitalized (3rd-generation) systems: Street-level bureaucracies have given way to system-level bureaucracies to transform administrative discretionary power [5, 14], online tools are used as channels for government agencies to receive feedback from citizens [9], and social media as a form of managing public relations [15].

The underlying framework however remains the same: the model of politics, legislation through elected representatives, the model of public revenue and expenditure, the culture of red tape, etc. Stemming from times when officialdom and bureaucracy, democracy and parliamentarianism, the social state and worker's unions, VAT and the fiat monetary system, were radically novel ideas enabled and supported by a society churned up by the transition from agrarian to industrial economy, these established cultures and institutions continue to withstand the possibilities of 4th generation technology. 
Existing culture in this domain implies limits, which impact the way technology can be applied. In the domain of public administration, for example, Lenk distinguishes between three types of processes [13]: recurrent and well-structured processes which can be automated on the one side, and individualized decision-making as well as negotiation processes, which can be supported by IT, but not automated, on the other. The first two types of processes have largely been digitalized (automated decisions, screen / system level bureaucracy; [cf. 5]), but further pushes for modernization within the limits of digitalization lead to controversial ideas and developments such as the use of data mining techniques for analysing the sentiment of the governed subjects. While such ideas (contemporarily promoted using the buzzword Big Data) do not change the established paradigms of societal governance, they bear the immanent risk of preventing the evolution of history (Riedl: "Geschichtsbremse" - "history drag" [25]) by reinforcing existing power relations of governance systems beyond repair. I've raised the manifold issues of digitalized government technology previously $[20,23]$, pointing out that such technology is costly, unsustainable and discriminative in the worst case, while in the best case the dependency on such systems leads to the emergence of a neo-feudal system in which controllers of the digitalized services have the upper hand.

\subsection{Governance informatization}

Unlike digitalization of governance, governance informatization for now exists only on a level of conceptual studies and proof-of-concept experiments. Outlined below are three concepts, which base on the paradigm of informatization - i.e. embrace the principles of networked co-creation of open, accessible files as a matter of controlling systems of public governance. These concepts are Governance Informatization [22], Liquid Democracy [21], and the Quantum Budget.

\section{Governance Informatization}

Initially termed "self-service government" [22], then "informating governance" [19], the concept of Governance Informatization (GI) bases on the assumption that a system of distributed files containing jurally relevant facts (such as certificates of education, driving permits, etc.) could serve as a source to determine one's eligibilities in a given (jurally relevant) situation. The principles of GI are inspired by Jellinek's systemisation of jural eligibilities into the system of subjective public rights [10], which describes one's position in a jurally relevant situation through the involved individuals' jural status. This system would allow subjects to co-create governance by altering the contained data, whereby access to data would be regulated through a dynamic fine-grained access control system.

\section{Liquid Democracy}

Liquid Democracy (LD) is a way of making collaborative decisions through a system, in which interpersonal relations of trust are digitally stored, and thus reflect an ever-changing (hence, "liquid") network, through which communal decisions in matters such as legislation, appointments of representatives, public spending, etc., can be made [21]. From a perspective of democratic theory, this way of collaborative decision making has been found superior to other forms of direct democracy [4]. The concept of LD is complementing Governance Informatization, in terms that it allows the engineering of a general-purpose technical system able to endure future changes of the hosted real-world systems of governance [21]. 


\section{Quantum Budget}

With the combined power of Governance Informatization and Liquid Democracy, a new approach to public revenue and public spending can be realized, where taxes would not be transferred to a central authority, but instead remain in the possession, although outside of control of taxpayers. Taxes owed by a subject to the community would instead be automatically (i.e., formula-based - cf. the key-lock paradigm from [22]) locked according to valid legislation, and would be used for purposes of public funding when required. This would open new ways to look at taxation and public funding, which however yet need to be further explored.

\section{Discussion - a new spectre that haunts the world?}

Expecting institutions of public governance to give up their rubber stamps in order to voluntarily give way to new paradigms of governing the common good, would be naïve - it doesn't fit the lifecycle of these bureaus, as Downs described it [7]: bureaus (as do other types of organisations such as churches, corporations, etc.) follow the ambition to expand their territory and influence, take over other bureaus and their resources, and increase their revenue. For satisfying these ambitions, digitalization is a suiting approach, as it positively addresses performance in terms of effectiveness, efficiency, and sometimes economy, without meddling with the overall architecture of institutions that make up public governance. Digitalization thus turns into a welcomed tool to demonstrate modernization through technology, without endangering the hegemony of the bureaucracy.

Informatization, on the other hand, is defined amongst others by its reliance on co-creation, which implies accessible (open, transparent) files and communication protocols, and comes with an inherent attitude that warmly embraces self-service - an important added value of informatization is thus the elimination of the middle-man. To this end, informatizing public governance would openly challenge strongly rooted models and narratives of public governance, which crucially rely on a service-based paradigm of operation (officers to issue permits, municipal commissions to decide on public funding, electoral committees to govern elections, legislative assemblies to pass laws, etc.).

Was it yet socialism, which haunted the entrenched European aristocratic and cleric establishment during the last decades of the second industrial revolution, it is now the pressing informatization that calls for transformation in all segments of society. What both spectres of change have in common, is the hope for new opportunities for emerging generations whose appetites the entrenched systems fail(ed) to satisfy. Prominent proponents of organizations, which embraced the new opportunities of informatization with success, are corporate giants such as Google, Uber, or Tencent, and non-profit social service providers such as Mozilla, the W3C, Wikimedia, or Wikileaks.

Gathering knowledge for building towards the transition to actually-existing informatized governance is the duty of science and engineering. This task is to be approached by developing theories, concepts, models, and laboratory experiments, which further explore the implications and potentials of governance informatization as the next step in transforming governance to a level beyond bureaucracy. 


\section{References}

[1] ALBROW, M. 1970. Bureaucracy. Macmillan.

[2] BANŪ-MŪSĀ, AHMAD IBN MŪSÁ IBN ŠĀKIR and ḤASAN IBN MŪSÁ IBN ŠĀKIR 1979. The book of ingenious devices. Reidel.

[3] BEVIR, M. 2009. Key concepts in governance. SAGE.

[4] BLUM, C. and ZUBER, C. I. 2016. Liquid Democracy: Potentials, Problems, and Perspectives: Liquid Democracy. Journal of Political Philosophy. 24, 2 (Jun. 2016), 162-182.

[5] BOVENS, M. and ZOURIDIS, S. 2002. From Street- Level to System- Level Bureaucracies: How Information and Communication Technology is Transforming Administrative Discretion and Constitutional Control. Public Administration Review. 62, 2 (Dec. 2002), 174-184.

[6] BUNDESMINISTERIUM FÜR BILDUNG UND FORSCHUNG 2013. Zukunftsbild "Industrie 4.0."

[7] DOWNS, A. 1967. Inside bureaucracy. Little, Brown.

[8] GRETZEL, U., WERTHNER, H., KOO, C. and LAMSFUS, C. 2015. Conceptual foundations for understanding smart tourism ecosystems. Computers in Human Behavior. 50, (Sep. 2015), $558-563$.

[9] HARTMANN, S., MAINKA, A. and STOCK, W. G. 2017. Citizen Relationship Management in Local Governments: The Potential of 311 for Public Service Delivery. Beyond Bureaucracy. A.A. Paulin, L.G. Anthopoulos, and C.G. Reddick, eds. Springer International Publishing. 337-353.

[10] JELLINEK, G. 1905. System der subjektiven öffentlichen Rechte [System of subjective public rights]. JCB Mohr (P. Siebeck).

[11] KATSIKAS, S. K. and GRITZALIS, S. 2017. Digitalization in Greece: State of Play, Barriers, Challenges, Solutions. Beyond Bureaucracy. A.A. Paulin, L.G. Anthopoulos, and C.G. Reddick, eds. Springer International Publishing. 355-375.

[12] KEATS, J. 2009. The Mechanical Loom. Scientific American. (Aug. 2009).

[13] LENK, K. 2012. The Nuts and Bolts of Administrative Action in an Information Age. Innovation and the Public Sector. (2012), 221-234.

[14] MAKOWSKI, G. 2017. From Weber to the Web... Can ICT Reduce Bureaucratic Corruption? Beyond Bureaucracy. A.A. Paulin, L.G. Anthopoulos, and C.G. Reddick, eds. Springer International Publishing. 291-312.

[15] MAMBREY, P. and DÖRR, R. 2011. Local Government and Social Networking Technologies in Germany: The Example of Twitter. Proceedings of the International Conference for EDemocracy and Open Government (Krems, 2011). 
[16] OXFORD ENGLISH DICTIONARY 2010. computerization, n. Oxford English Dictionary.

[17] OXFORD ENGLISH DICTIONARY 2010. digitalization, n.2. Oxford English Dictionary.

[18] PAULIN, A. 2017. Data Traffic Forecast in Health 4.0. Health 4.0: How Virtualization and Big Data are Revolutionizing Healthcare. C. Thuemmler and C. Bai, eds. Springer International Publishing. 39-60.

[19] PAULIN, A. 2016. Informating Smart Cities Governance? Let Us First Understand the Atoms! Journal of the Knowledge Economy. (Apr. 2016).

[20] PAULIN, A. 2016. Technological Ecosystems' Role in Preventing Neo- Feudalism in SmartCity Informatization. Proceedings of the 25th International Conference on World Wide Web Companion (Montreal, Canada, Apr. 2016).

[21] PAULIN, A. 2014. Through Liquid Democracy to Sustainable Non-Bureaucratic Government - Harnessing the Power of ICTs for a novel form of Digital Government. eJournal of eDemocracy and Open Government. 6, 2 (2014).

[22] PAULIN, A. 2013. Towards Self-Service Government - A Study on the Computability of Legal Eligibilities. Journal of Universal Computer Science. 19, 12 (Jun. 2013), 1761-1791.

[23] PAULIN, A. 2015. Twenty Years After the Hype: Is e-Government doomed? Findings from Slovenia. International Journal of Public Administration in the Digital Age. 2, 2 (32 2015), 121.

[24] RAYMOND, E. S. 1999. The Cathedral \& the Bazaar: Musings on Linux and Open Source by an Accidental Revolutionary. O'Reilly Media.

[25] RIEDL, R. 2016. Big Data - schnell erklärt. SocietyByte - Wissenschaftsmagazin des BFHZentrums Digital Society.

[26] SCHABER, W. 2004. Hellbrunn Schloss, Park und Wasserspiele. Schloss Hellbrunn.

[27] SCHUPPAN, T. and KÖHL, S. 2016. Verwaltung 4.0: Modernisierungsrelevant oder alter Wein in neuen Schläuchen? Verwaltung \& Management. 22, 1 (2016), 27-33.

[28] SHANKEN, E. A. 2000. Tele-Agency: Telematics, Telerobotics, and the Art of Meaning. Art Journal. 59, 2 (2000), 64.

[29] SMIL, V. 2005. Creating the Twentieth Century. Oxford University Press.

[30] SÖDERSTRÖM, O., PAASCHE, T. and KLAUSER, F. 2014. Smart cities as corporate storytelling. City. 18, 3 (May 2014), 307-320.

[31] THUEMMLER, C. and BAI, C. eds. 2017. Health 4.0: How Virtualization and Big Data are Revolutionizing Healthcare. Springer International Publishing. 